\title{
Case Study: Public Transport Management System for Kousba - Chekka Roadway
}

\author{
Georges Najib, Fawaz Samer, Karam George \\ University of Balamand \\ Kelhat, Koura, Lebanon \\ ngeorges@balamand.edu.lb; samer.fawaz@fty.balamand.edu.lb; georges.karam@std.balamand.edu.lb
}

\begin{abstract}
Two of the main reasons of traffic congestion in Lebanon are the high number of private vehicles and the absence of a decent public transportation system. While the problem regarding the unavailability of the public transportation system can be related to the government, the high number of private cars can be explained and tackled from different angles. This study aims to present a full bus system analysis and design as a solution for various problems regarding the transportation sector. 1000 person completed a survey asking about the existing buses, and their willingness to use a decent bus system; results showed that $73 \%$ of the voters would take this advanced bus. That means a reduction of 525 cars/ hour after installing 7 buses on the Kousba - Chekka road. This amount of cars replaced by buses also implied a decrease of noise pollution by $2 \mathrm{~dB}$, and a significant decrease of accidents. It is concluded that a reliable bus system should be implemented.
\end{abstract}

Keywords: Public Transport, Bus System, Lebanon, Traffic congestion

\section{Introduction}

As is the case in such situations, the Land Transport Sector found itself greatly jeopardized by the 1975 Lebanese Civil War. Aside from war-related issues, Lebanese Transportation history reveals an even more disturbing problem. Indeed, the average Lebanese's constant reliance on private cars, coupled with uncoordinated public transportation, has led to an increasing rate in congestion and severe trafficking problems. As a consequence, motor transportation increased, leading to severe, borderline unacceptable, motorcycle-related mortality rates. Thus this project is presented with high hopes that it might at least contribute to future improvements.

The road studied is located in the Koura area connecting Kousba and Chekka, and passing by Kfaraaka, Kfarsaroun, Amioun and Kfarhazir.

\section{Methodology}

Data collection was done through a survey where people have to answer a questionnaire that consists of 15 questions that help assess and estimate the ridership for a public transport system. Data was collected from field visits to local private bus operators to better understand the volume of public bus users and the way public busses are operated. A traffic count was performed to assess the traffic volumes.

An aerial view or a bird eye view was obtained in order to locate the optimal points for bus stops such that no public bus user will have to walk more than $200 \mathrm{~m}$ to reach the nearest bus stop.

The data collected was then analysed in order to design the bus cycles and system.

\section{Problems Related to the Transportation Sector in Lebanon}

\subsection{Accidents}

A report posted by the Lebanese Red Cross in 2014 shows the distribution of road accidents according to sex, age group and vehicle type and it reveals a very high number of deaths (229) and injured (14516). Young people are the most affected by road accidents and that should be a major concern for the country.

According to latest data collected by YASA Lebanon (Youth Association for Social Awareness), the number of accidents in Lebanon is very high. In 2012 the number of accidents reached 4804 with 595 deaths and 6697 injured. 
In the north district, where this study is done, $16.01 \%$ of the accidents occur with 117 death and 997 injuries.

Classifying the injured and dead, it is found that the highest percentage of losses is the age group between 15 and 29, and that is a major problem to the country since this category represents the working base and the future of Lebanon. This also implies that a lack of transportation awareness in schools, universities and families, and a lack of governmental supervision and strict measures against violations are the main problems. Comparing Lebanon to other countries, it was found that Lebanon has a high death rate.

Lebanon is the 84th country in the ranking with a rating of 17.2 dead / 100000 humans.

Accidents in Lebanon are due to different problems:

- Bad condition of the roads

- Reckless and illegal driving

- Absence of a national transportation code

- The high number of passenger cars

Accidents in Lebanon are not only causing life losses but also economical and financial losses. Choueiri et al. (2010) showed that accidents victims spend an average of 1.76 working days in hospitals costing $600 \$ /$ day. Assuming 3400 accidents per year, the treating of accidents injuries costs about $3.6 \mathrm{M} \$$ /year. They also showed that accidents cost the national economy about $1.5 \%$ of the GDP (the gross domestic product).

\subsection{Traffic Congestion}

As mentioned before, passenger cars in Lebanon consist of $86 \%$ of all types of vehicles. A study done in 2005 about the estimated growth of vehicles in Lebanon estimated an increase to 1200 thousands vehicle in 2011. Thus the real number in 2011 turned out to be 1400 thousands vehicles and a new curve was drawn. This red curve estimated a growth to 1750 thousands vehicles in 2015 .

In 2010, the ratio of cars to person in Lebanon was about 1 car to every 3 person. In 2012 this ratio increased to 434 cars per 1000 person. And now in 2015, this number is estimated to be 500 cars for every 1000. This ratio is one of the highest in the world and the main reason of traffic congestion. Choueiri et al. (2010) discussed that in 2010 two third of the daily motorized trips were made by passenger cars and this ratio was estimated to increase by 2016 .

\subsection{Noise Pollution}

Noise pollution is one of the most underrated and harmful problem related to the transportation sector. Acceptable noise levels are exceeded on most of Lebanese roads. The figure below shows maximum acceptable levels of noise.

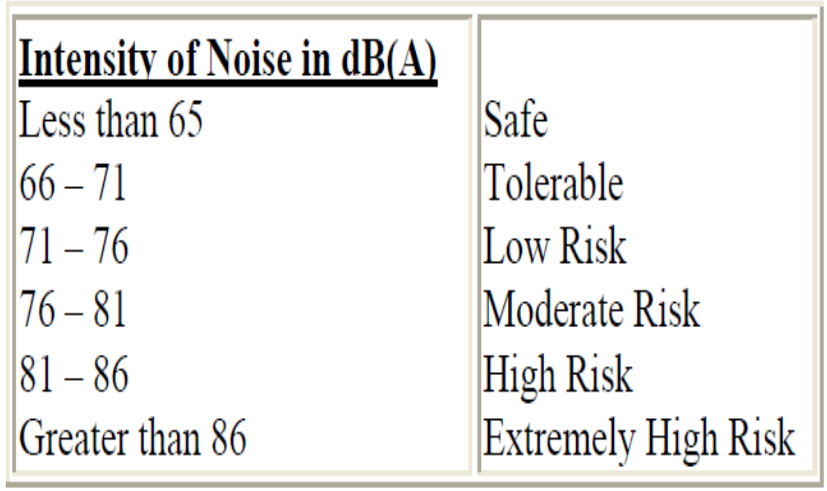

Fig. 1: Range of noise level.

The existing noise level on the road was determined according to the $\mathrm{L}_{10}$ nomograph. The total number of cars calculated is $1409 \mathrm{veh} / \mathrm{hr}$ at a speed of $60 \mathrm{~km} / \mathrm{hr}$ with heavy trucks counted as $9 \mathrm{veh} / \mathrm{hr}$. The value of the noise generated from the road was found to be $75 \mathrm{db}$ for trucks and $71 \mathrm{~dB}$ for passenger cars and medium trucks. With $\Delta=4 \mathrm{~dB}$ as the difference between the two previous values we get a noise level of $75+1.5=76.5 \rightarrow$ Moderate risk. 


\subsection{Air Pollution}

A report published by the ministry of environment in Lebanon in 1994 revealed that the transportation sector is responsible of $94 \%$ of $\mathrm{CO}$ emissions and $40 \%$ of $\mathrm{CO}_{2}$ emissions. The report explained that one of the reasons of this high percentage is the high number of passenger cars and the bad conditions of the vehicles (older than 14 years). Statistics done by the MoE 2000 (Lebanon's Second National Communication to the UNFCCC, 2011), show that the transportation sector in Lebanon is responsible for over $21 \%$ of total emissions.

The Australian greenhouse office produced a report where it shows how different vehicles produce different quantities of GHGs. This report illustrates the different quantities of gas emissions as Kilograms per person per kilometer. What was stated was that a bus produces $0.003 \mathrm{~kg} /$ person of gases, which is much less than a normal passenger car which produces $0.32 \mathrm{~kg} /$ person. It can be concluded, that a decent bus system can effectively reduce the GHG emissions in Lebanon.

\section{Data Collection}

\subsection{AADT Calculation}

After collecting data from many manual traffic counts on various timings, it was found appropriate to use tables made by the NRA (national road authority) in Ireland. The average annual traffic volumes were found to be: $10142 \mathrm{v} / \mathrm{d}$ upstream, and 9026v/d downstream. So the total volume in both directions would then be 19168v/d.

It was also needed to calculate average daily volume for passenger cars, medium trucks and heavy trucks separately for this study. The numbers were found to be:

- 16833 Passenger cars / day

- 1700 medium trucks / day

- 212 heavy trucks / day

\subsection{Questionnaire Analysis}

In order to better understand the situation of the transport sector and the opinion of the general population toward the Kousba - Chekka road, a questionnaire was distributed to a sample of 1000 people from the Kousba - Chekka area. The answers to the questionnaire were then collected, managed, and analysed.

The delivery of the sample seems to endorse the results obtained from other studies that were performed by others. $51 \%$ of the investigated people were males and $49 \%$ were females. The distribution by age of the sample demonstrates that a big percentage of the road users are under the age of 25. This implies that the complications resulting from unawareness could be treated for the most part through education in schools and universities.

The average number of family members was estimated to be 4 members. To complete this analysis the numbers of cars per family was needed. The average number of cars per family was estimated to be 2 cars. The ratio of vehicles per capita is estimated to be 2 . A previous study done in 2012 by Nation Master ranked Lebanon the forty second country in the world with 434 vehicles per 1000 and the ratio increased since then.

One of the main parameter in the study is the destination of travellers in order to compute the actual number of road users. What was important is only destinations related to this study the following results are obtained:

The fuel cost of car users per day was found to be 24191.02 L.L. Assuming 5 working days per week, transportation costs are 483820.4 L.L. per month. This number is $80 \%$ of the minimum salary in Lebanon set by the government, which is a very high percentage. This huge amount is very unreasonable in this country. Public transportation is the best solution (bus system).

Passenger cars represent the highest percentage of transportation vehicles in Lebanon. Buses, taxis, motorcycles, shared taxis, and bikes represent the alternative transportation mode. Asked about their alternative, people chose taxis $(53 \%)$ as their favourite alternative, which is also categorized as a private vehicle. This implies that most of Lebanese citizens have this mentality of traveling using only passenger vehicles. Buses are ranked second with $21 \%$. Comparing the costs between a taxi and an existing bus (Estfan) on the Kousba - Chekka road, taxis take an average of 20000 while bus charges 2000 that means only $10 \%$ of taxis. Thus, money is not the problem that is preventing people from taking the bus. 
Table 1: Traveller's destination.

\begin{tabular}{|c|c|}
\hline Destination & Number of Individuals \\
\hline Amioun & 128 \\
\hline Kousba & 86 \\
\hline Kfarhazir & 23 \\
\hline Kfarsaroun & 5 \\
\hline Chekka & 49 \\
\hline Kfaraaka & 1 \\
\hline Jbeil & 31 \\
\hline Batroun & 50 \\
\hline Beirut & 46 \\
\hline & 419 \\
\hline & $42 \%$ \\
\hline
\end{tabular}

A study done by transportation experts in seven different countries, with Beirut one of them presented important data. 1229 surveys were analyzed on students from seven different countries: China, Indonesia, Japan, Lebanon, Netherlands, Taiwan, and USA. 271 observations were done in Beirut. It was observed that the city of Beirut has the highest car users' \% (89.7), highest car user and owner \% (85.6) and the second lowest public transportation usage \% (17.4). This study explained that the main reasons why people do not take buses are:

- Drivers drive carelessly

- People feel unsafe riding public transport

- The service is unreliable

- People get annoyed by long waiting times at stops

- The service doesn't cover the city area well

It is clear that existing bus systems in Lebanon are not preferred by Lebanese. According to the survey, the rating of the buses in Lebanon was found to be low. The people gave the current public transport system a rating of approximately 4/10. This confirms that the bus system in Lebanon is poor and needs improvement.

This improvement can be done by:

- New buses that assures safety and comfort

- Hiring legally qualified and responsible drivers

- Professional management of time schedule and bus cycles

- Locating bus stops in a way that covers most populated areas

People were asked if they would take such advanced buses if introduced to the road, the majority got impressed with the idea.

$73 \%$ out of 893 voters would take the bus. This reduction will help in lowering the traffic, increasing road life and maintenance costs, and reducing pollution and accidents. $73 \%$ of 16833 passenger cars are 12288 passenger cars / day removed from the road.

\section{Bus System Design}

\subsection{Bus Stops}

It is globally recommended in the design standards for populated area that the maximum reaching distance is $400 \mathrm{~m}$. On a satellite view of the road, circles of a $200 \mathrm{~m}$ radius in residential areas were drawn to locate the bus stops. 


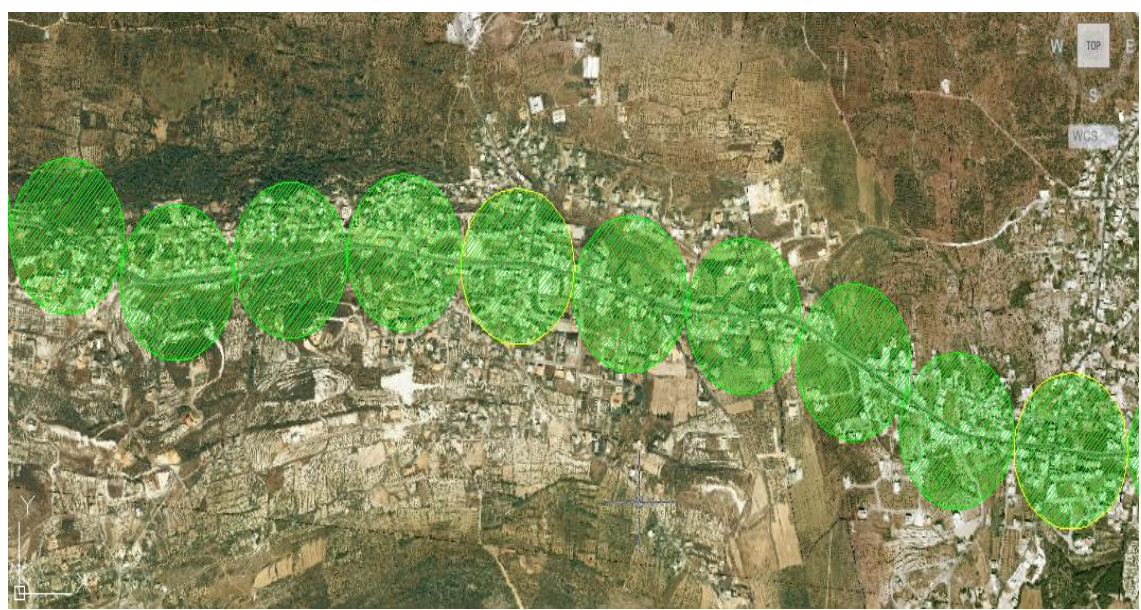

Fig. 2: Locating bus stops.

According to the guidelines set by the Metropolitan Transit Development Board in San Diego, there are two types of bus stops. The two types are: Bus stops near intersections and bus stops at Midblock.Each equation should be presented on a separate line from the text with a blank space above and below.

\subsubsection{Bus Stops near Intersections}

The bus stop longitudinal distance is $20 \mathrm{~m}$. A minimum of $12 \mathrm{~m}$ no parking zone should be provided at the end of the bus stops to ensure a safe and proper bus service. The no parking zone between the bus and the intersection varies according to surroundings.

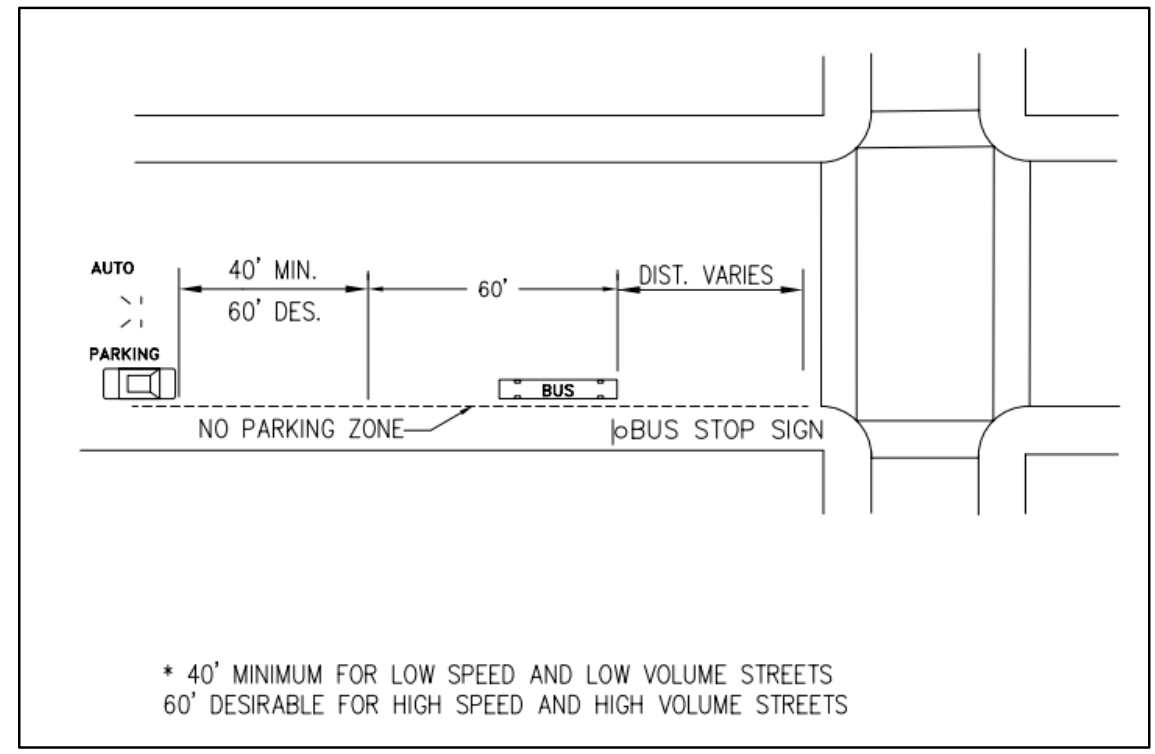

Fig. 3: Bus stops near intersections.

\subsubsection{Bus Stops at Midblock}

In this case, the no parking zones should be minimum provided at $12 \mathrm{~m}$ at both ends. 


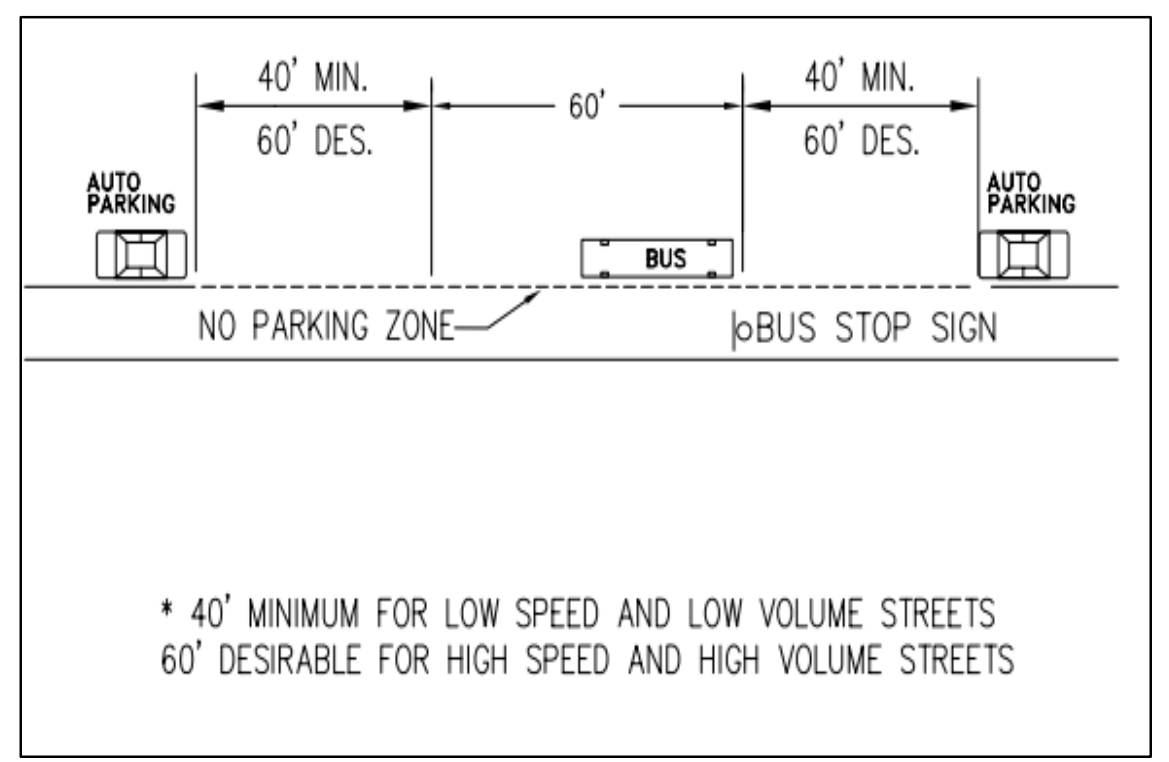

Fig. 4: Bus stops at midblock. to tail).

The Road Service Transportation Unit in UK recommends that opposite bus stops minimum separation is $36 \mathrm{~m}$ (tail

\subsection{Bus Cycle}

A public transportation expert, Christopher MacKechnie, proposed a manual calculation procedure to compute bus cycles. The procedure is as follows:

i. Placement of the bus route: The studied bus route is from Kousba to Chekka passing by Kfaraaka, Kfarsaroun, Amioun and Kfarhazir. The total cycle is from Kousba to Chekka and back.

ii. Locating time points: Time points are the most important positions of buses. Start and end are the first 2 time points. Other time points are taken as bus stops responsible of more than $10 \%$ of the total boarding. In this case time points were located as follows:

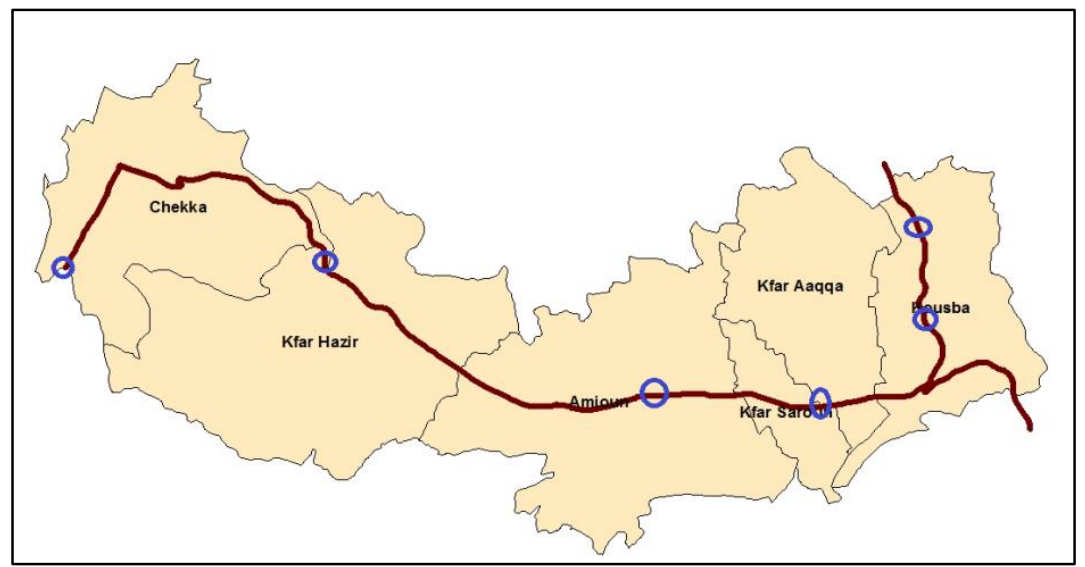

Fig. 5: Locating time points.

Start and end points in Kousba and Chekka respectively were taken as time points. Bus stops near intersections in Kousba, Kfaraaka, Amioun and kfarhazir were also considered as time points.

iii. Bus cycle calculation: Cycles are calculated with 2 methods and the maximum value is used in design. 
The first method is to drive the road from start to end with a speed less than the maximum by $10 \mathrm{Km} / \mathrm{hr}$ and then multiply this number by a loss factor $=1.3$. In this case, driving the road from Kousba to Chekka recorded a time of 18.35 $\min$.

Multiplying by 1.3 , the computed number of 1 direction drive is 23.855 min $\rightarrow$ use 24 min. The second method is to calculate the time required to drive between each time point and add them which resulted in 20.63 minutes.

Comparing the 2 cycles, 23.855 is larger than $20.63 \rightarrow$ Use $24 \mathrm{~min}$. According to the procedure, it is desirable that cycle times are multiples of 30-60 $\mathrm{min}$ so the total cycle is taken as 1 hour $(30 \mathrm{~min}+30 \mathrm{~min})$.

Referring to data collected from local bus operators, buses will be operated on a 15 min frequency. A total of 5 buses will be needed for optimum productivity.

Checking if these buses will serve passengers at peak hours:

Calculation:

From the questionnaire analysis, $73 \%$ agreed to use the advanced bus system and that is a reduction of 12288 passenger cars/ day. Taking an average of 2 persons / car, that makes a total of 24576 riders / day. From the questionnaire analysis, only $42 \%$ of these road users benefit from this bus system according to their destination (Kousba, Kfarsaroun, Kfaraaka, Amioun, Kfarhzir, Chekka). This will result to a reduction to 430 bus riders / cycle.

The bus chosen for this design has a maximum capacity of 87 bus riders. Thus, the number of buses required is 5 buses (in peak hours) so the initial assumption is correct.

\section{Project Impact:}

The high number of accidents on this road will be reduced significantly. Traffic jam will also be reduced due to the high decrease of passenger cars. Noise pollution will be reduced to $75.5 \mathrm{db}$ which is classified as low risk.

According to the graph, the amount reduced of $\mathrm{mg} \mathrm{GHG:}$

Adding 5 buses will emit $0.003 \times 5=0.015 \mathrm{~kg} / \mathrm{km}$, however, omitting 12288 passenger cars will lead to a decrease of 0.16 $(\mathrm{kg} /$ pers $/ \mathrm{km}) \times 2$ (pers) x 12288 (cars) $=3932 \mathrm{~kg} / \mathrm{km}$

Air pollution will be considerably decreased

\section{Conclusion \& Recommendations:}

Several problems on the Kousba - Chekka road related to the transportation sector are considered as a threat to life and to the safety of citizens. Accidents, noise pollution, air pollution and traffic jam can be all solved by installing a reliable bus system. With a positive feedback from the population regarding the installation of an advanced public transportation system, along with the current bad conditions of the existing bus systems, this project, along with its innovative futuristic insights, is indispensable for better transportation services. Public transportation is a major solution for problems related to the transportation sector, but it is not enough. More serious steps should be taken into consideration such as:

- Raising awareness in schools and universities about traffic laws and transportation

- Traffic law enforcement

- More investment in the transport sector

\section{Acknowledgements}

The authors would like to express gratitude to Ramy Abdul Kader and Georges Bejjani who were of great assistance in writing this paper and in data collection.

\section{References}

[1] P.F. Belgiawan, J. Walker, J. Schmöcker,S. Fujii, T. Lee, M. Abou-Zeid, and D.F. Ettema, "Car ownership motivations among undergraduate students in china, indonesia, japan, lebanon, netherlands, taiwan, and USA," Transportation, vol. 41, no. 6, pp. 1227-1244, 2014.

[2] GIS Center, "Urban Noise Mapping: The Case of the City of El-Mina, North Lebanon," University of Balamand. 
[3] National Roads Authority. Project Appraisal Guidelines [Online]. Available: http://www.tii.ie/tiilibrary/strategic-planning/project-appraisal-guidelines/Unit-16.2-Expansion-Factors-for-Short-PeriodTraffic-Counts.pdf

[4] University of Purdue. Highway Noise Impacts Assessment and Evaluation [Online]. Available: https://engineering.purdue.edu/ ce561/classnotes/Chapter\%2012.pdf

[5] Darnell \& associates. (2006, October 4). Bus Stop Design Guidlines [Online]. Available: http://www.omnitrans.org/about/reports/pdf/BusStopGuidelines_10-04-06.pdf

[6] C. Mackechnie. Designing Bus Routes and Schedules [Online]. Available: http://publictransport.about.com/od/Transit_Planning/a/How-Bus-Routes-And-Schedules-Get-Planned-Part-IPlacement-Of-Bus-Route.htm

[7] Red Cross Lebanon. (2008). [Online]. Available: www.redcross.org.lb

[8] YASA. (2009). [Online]. Available: www.yasa.org

[9] Lebanese Interior Security Forces. (2016). [Online]. Available: www.isf.gov.lb 